\title{
Prevention of diabetic foot ulceration: new evidences for an old problem
}

\author{
Luigi Uccioli
}

Received: 13 February 2013/Accepted: 12 March 2013/Published online: 26 March 2013

(C) Springer Science+Business Media New York 2013

Diabetic patients have a $15 \%$ lifetime risk of developing a foot ulcer and have a $15 \%$ risk of developing a lower extremity amputation. Amputation is preceded by nonhealing foot wounds in $85 \%$ of cases [1]. The ulcerated diabetic foot may be considered the complication of the peripheral neuropathy (PN) and/or peripheral arterial disease (PAD).

Several studies showed that diabetic patients with PN are at risk of foot ulceration and indeed true neuropathic ulceration is found in almost $50 \%$ of the patients with a new foot ulcer according to eurodiale [2]. Those with PAD are at risk of foot amputation and ischemic lesions are found in the other $50 \%$ of patients with foot ulceration. However true ischemic ulcers are observed only in $15 \%$ of cases. The remaining $35 \%$ have both PAD and PN present at the same time: neuroischemic ulcers [2].

Many factors may be responsible of the appearance of an ulcer in those patients: intrinsic factors related to the progressive appearance of limited joint mobility, soft tissue stiffness, early and persistent windlass mechanism [3] presence of structural foot deformities as hammer toes, hallux valgus or pes cavus. Metatarsal heads are prominent. This results in increased pressure underneath the metatarsal heads, which will make the plantar forefoot more susceptible to breakdown, also because patients are unaware of this abnormal pressure because of the neuropathy.

However, there are also other contributing factors leading to diabetic foot ulcer formation, such as arterial occlusive disease, foot trauma, history of previous amputations, and callus due to the increased plantar pressures caused by

L. Uccioli $(\bowtie)$

Department of Internal Medicine, University of Rome Tor

Vergata, Viale Oxford 81, 00133 Rome, Italy

e-mail: luccioli@yahoo.com abnormal mechanical loading of the foot [4]. A sequence of events has been described in the chain of events that bring to a new foot ulceration: neuropathy is the predisposing condition, trauma is the precipitating factor [5].

While neuropathic ulcer may spontaneously heal whenever the offloading is guaranteed, the ischemic one has no chance to heal unless a revascularization, either traditional but even better with peripheral angioplasty [6].

A recent study showed that male gender and PN are predictors of diabetic ulcers and amputation, and according to the literature there is an increasing risk of amputation in those patients with both PN and PAD [7].

Prevention starts selecting the patients at risk. Very simple tests may be applied. PN may be highlighted by semiquantitative tests such as monofilaments. Different protocol may be utilized with 3-10 points to test, and according to the number of insensitive points the patient is defined as neuropathic or not [8].

Another method commonly utilized is the definition of the vibratory perception threshold. In this case, values more than 25 indicate the presence of PN with an increased risk of foot ulceration [9]. Patients with PAD are categorized by using the ankle brachial index $(\mathrm{ABI})$; values $<0.9$ indicate the presence of PAD with an increased level of gravity when values $<0.5$ are recorded (critical limb ischemia) [10].

Once defined the presence of PN, PAD, the presence of foot deformities, limited joint mobility, recorded previous history of ulceration, and/or amputation, it is possible to classify the patients in the appropriate risk categories from class 0 without risk to class 3 at very high risk of foot ulceration [11].

The main elements of a prevention program are three: education, podiatric cures, regular use of protecting shoes and insoles. 
The patients should learn how to substitute a lost sensations (related to PN) with another one: i.e., test with the hands the presence of eventually extra bodies (stones, coins) inside the shoes or test with the elbow the water temperature before to wash the feet. In addition, they should adopt some preventive precautions such as never walk barefoot mainly on the sand. Patient education is an integral part of prevention work and has the purpose of changing the criteria of self-management of the patient and to improve adherence to health claims related to foot care, such as the use of footwear.

Although many authors believe that preventive education is of significant importance in preventing foot lesions caused by external precipitating factors and those caused by improper self-treatment or neglect a recent meta-analysis showed that there is no evidence that the only patient education has effectiveness in achieving a clinically relevant reduction of ulcers and amputations [11].

Several studies demonstrated that at risk patients may take advantage in being included in a surveillance program driven by a team. It may guaranteed, beside the monitoring of blood glucose and the specific educational reinforcement, the necessary podiatric treatments, able to actively reduce by itself the ulceration risk: callus removal allows to achieve a $30 \%$ reduction of the hyper load under the metatarsal heads [12]. In certain cases, patients with rigid deformity and limited joint mobility that cannot be managed by the usual care (callus removal and offloading) should be evaluated for a certain type of prophylactic foot surgery to correct the structural deformities [13].

The third factor able to reduce the risk of foot ulceration is the regular use of preventive shoes and insoles that may $50 \%$ cut hyperpressures under the foot.

Shoes are thought, designed, and constructed to allow comfortable progression action while maintaining as much as possible the biomechanics of a physiological gait. Several studies support the belief that inappropriate footwear causes ulceration. [14]. While the role of specific shoes has been proven in secondary prevention with a significant reduction of relapses in the group treated by therapeutic shoes and customized orthesis [15], its role in primary prevention is still not definitively proven.

Recently, a paper by Rizzo et al. [16] has shown a protective effect of shoes and insoles in a group of patients with a risk score $\geq 2$, which included both patients in primary and secondary.

In conclusion, a strict follow-up driven by a team that may guarantee specific education, intensive podiatric care, and the use of orthesis and shoes can reduce the incidence of foot ulcers in diabetic patients who are at high ulcerative risk.

\section{Conflict of interest none.}

\section{References}

1. G. Apelqvist, Diagnostics and treatment of the diabetic foot. Endocrine 41, 384-397 (2012)

2. L. Prompers, N. Schaper, J. Apelqvist, M. Edmonds, E. Jude, D. Mauricio, L. Uccioli, V. Urbancic, K. Bakker, P. Holstein, A. Jirkovska, A. Piaggesi, G. Ragnarson-Tennvall, H. Reike, M. Spraul, K. Van Acker, J. Van Baal, F. Van Merode, I. Ferreira, M. Huijberts, Prediction of outcome in individuals with diabetic foot ulcers: focus on the differences between individuals with and without peripheral arterial disease. The EURODIALE Study. Diabetologia 51, 747-755 (2008)

3. E. D'Ambrogi, L. Giurato, M.A. D'Agostino, C. Giacomozzi, V. Macellari, A. Caselli, U. Uccioli, Contribution of plantar fascia to the increased forefoot pressures in diabetic patients. Diabetes Care 26, 1525-1529 (2003)

4. N. Singh, D.G. Armstrong, B.A. Lipsky, Preventing foot ulcers in patients with diabetes. JAMA 293, 217-228 (2005)

5. G.E. Reiber, L. Vileikyte, E.J. Boyko, M. Del Aguila, D.G. Smith, L.A. Lavery, A.J. Boulton, Causal pathways for incident lower-extremity ulcers in patients with diabetes from two settings. Diabetes Care 22, 157-162 (1999)

6. L. Uccioli, R. Gandini, L. Giurato, S. Fabiano, E. Pampana, V. Spallone, E. Vainieri, G. Simonetti, Long-term outcomes of diabetic patients with critical limb ischemia followed in a tertiary referral diabetic foot clinic. Diabetes Care 33, 977-982 (2010)

7. A. Moura Neto, D.E. Zantut-Wittmann, T.D. Fernandes, M. Nery, M.C. Parisi, Risk factors for ulceration and amputation in diabetic foot: study in a cohort of 496 patients. Endocrine. 2012

8. J.L. Richard, L. Reilhes, S. Buvry, M. Goletto, J.L. Faillie, Screening patients at risk for diabetic foot ulceration. A comparison between measurement of vibration perception threshold and 10-g monofilament test. Int. Wound J. 10, 1742-1748 (2012)

9. M.J. Young, J.L. Breddy, A. Veves, A.J. Boulton, The prediction of diabetic neuropathic foot ulceration using vibration perception thresholds. A prospective study. Diabetes Care 17, 557-560 (1994)

10. International Consensus on the Diabetic Foot (2009), http://www. iwgdf.org/. Accessed 1 Mar 2013

11. J.A.N. Dorresteijn, D.M.W. Kriegsman, W.J.J. Assendelft, G.D. Valk, Patient education for preventing diabetic foot ulceration (review). Cochrane Database Syst. Rev. 10 CD001488, D001488.pub4 (2012). doi:10.1002/14651858

12. M.J. Young, P.R. Cavanagh, G. Thomas, M.M. Johnson, H. Murray, A.J. Boulton, The effect of callus removal on dynamic plantar foot pressures in diabetic patients. Diabet. Med. 9, 55-57 (1992)

13. B. Rhim, L. Harkless, Can we stop problems before they arise? Semin. Vasc. Surg. 25, 122-128 (2012)

14. L. Uccioli, C. Giacomozzi, The role of footwear in the prevention of diabetic foot problems. A. Veves, et al., (eds) The diabetic foot: medical and surgical management. Contemporary Diabetes. doi:10.1007.978-1-61779-791-0_26

15. L. Uccioli, E. Faglia, G. Monticane, F. Favales, L. Durola, A. Alleghi, A. Quarantiello, P. Calia, G. Menzinger, Manufactured shoes in the prevention of diabetic foot ulcers. Diabetes Care 18(10), 1376-1378 (1995)

16. L. Rizzo, A. Tedeschi, E. Fallani, A. Coppelli, V. Vallini, E. Iacopi, A. Piaggesi, Custom made orthesis and shoes in a structured follow-up program reduces the incidence of neuropathic ulcers in high-risk diabetic foot patients. Int. J. Low Extrem. Wounds 11, 59-64 (2012) 